\title{
Subintimal shift as mechanism for side-branch occlusion in percutaneous treatment of chronic total occlusions with bifurcation lesions
}

\author{
Juan Luis Gutiérrez-Chico ${ }^{1,2}$ (D), Carlos Cortés ${ }^{3,4}{ }^{\oplus}$, Mohamed Ayoub ${ }^{5}$, \\ Bernward Lauer ${ }^{6}$, Sylvia Otto ${ }^{6}$, Bernd Reisbeck ${ }^{2}$, Manuela Reisbeck ${ }^{2}$, \\ Christian Schulze ${ }^{6}$, Kambis Mashayekhi ${ }^{5}$ (i)
}

\begin{abstract}
${ }^{1}$ Department of Cardiovascular Medicine, Ruijin Hospital, Shanghai Jiao Tong University School of Medicine, Shanghai, China; ${ }^{2}$ CardioCare Heart Center, Marbella, Spain; ${ }^{3}$ Miguel Servet University Hospital, Zaragoza, Spain; ${ }^{4}$ Hospital Clínico Universitario de Valladolid, Spain; ${ }^{5}$ Division of Cardiology and Angiology II, University Heartcenter Freiburg - Bad Krozingen, Germany; ${ }^{6}$ Jena University Hospital, Jena, Germany
\end{abstract}

\begin{abstract}
Background: The aim of this study was to describe the mechanism of subintimal shift (SIS), standardise diagnostic criteria and sensitise the interventional community to this phenomenon. The treatment of chronic total occlusions (CTO) by means of percutaneous coronary intervention (PCI) is complicated by bifurcation lesions involved in the CTO segment or adjacent to it. Extraplaque expansion of intracoronary devices during CTO PCI may extend the dissection plane over the bifurcation with the consequential side or main branch compression by an intimo-medial flap. This phenomenon is hereby described for the first time and named subintimal shift.

Methods: Experienced CTO operators from 3 international high volume centers for CTO PCI retrospectively searched their personal records for paradigmatic cases of SIS, summarising key features and proposing diagnostic criteria.

Results: The series comprised 7 demonstrative cases, illustrating SIS by intravascular imaging (2 cases) or indirect angiographic signs during CTO PCI (5 cases). Five cases were triggered by stent expansion, 1 by balloon inflation and 1 case was aborted after angiographic warning signs. In 4 cases, SIS resulted in total occlusion of a branch, refractory to ballooning whenever attempted. Four cases required bailout intervention and in 2 cases the branch was left occluded, resulting in a rise of cardiac markers.

Conclusions: Subintimal shift is a noteworthy complication in CTO bifurcations, potentially resulting in occlusion of the relevant side or even the main branch. Intracoronary imaging prior to stenting is recommended to understand the tissue planes. Some counterintuitive peculiarities of this phenomenon, like its refractoriness to ballooning, must be known by CTO operators for its efficient resolution. (Cardiol J 2023; 30, 1: 24-35)
\end{abstract}

Key words: percutaneous coronary intervention, coronary heart disease, chronic total occlusion, bifurcation lesion, complication

Address for correspondence: Prof. Juan Luis Gutiérrez-Chico, MD, PhD, FESC, FACC, Head of Interventional Cardiology, Ruijin University Hospital, 197, $2^{\text {nd }}$ Ruijin Road, 200025 - Shanghai, China, tel: +34 615 319370/+86 13501974021, e-mail: juanluis.gutierrezchico@ictra.es; Prof. Kambis Mashayekhi, MD, PhD, Deputy Medical Director, University Heart Centre Freiburg - Bad Krozingen, Division of Cardiology and Angiology, Südring 15, 79189 - Bad Krozingen, Germany, tel: +49 7633 402-2105, e-mail: kambis.mashayekhi@universitaets-herzzentrum.de

Received: 17.05.2021 Accepted: 4.07.2021 Early publication date: 7.07.2021

This article is available in open access under Creative Common Attribution-Non-Commercial-No Derivatives 4.0 International (CC BY-NC-ND 4.0) license, allowing to download articles and share them with others as long as they credit the authors and the publisher, but without permission to change them in any way or use them commercially. 


\section{Introduction}

Percutaneous coronary intervention (PCI) of chronic total occlusions (CTO) has recently experienced substantial technical progress and standardization, thus improving safety and procedural success rates [1]. Nonetheless, some specific anatomic scenarios, like bifurcations, still pose an additional challenge for CTO-PCI [2]. The incidence of CTO bifurcation lesions (CTOBFL) during PCI varies based on definition from $25 \%$ to $50 \%$ [2-5]. CTO-BFL are associated with lower procedural success and higher complication rates [2]. The loss of relevant side branches has an adverse impact on short- and long-term clinical outcome during CTO PCI $[4,6]$ by reducing major adverse cardiac event-free survival if it is not promptly and efficiently solved. Therefore, this complication should be avoided whenever possible.

One potential mechanism of occlusion is the extension of the dissection plane over a branch due to extraplaque balloon or stent expansion at a different segment of the bifurcation, thus resulting in a shift of the enlarged subintimal space from one segment of the bifurcation to another. We propose the term "subintimal shift" (SIS) for this phenomenon, which entails some interventional peculiarities that must be known by CTO operators to efficiently handle it.

Due to the relatively low recognition of SIS, the lack of a standard characterization and the missing awareness in current CTO registries so far, there are no data available for a systematic study of this phenomenon based on large CTO databases. Therefore, a short case series with representative SIS cases is hereby presented for the first time, aiming to describe the mechanism, propose a standard operational definition and discuss some interventional peculiarities of SIS in CTO-BFL.

\section{Methods}

Experienced CTO operators from three international centers (J.L.G.C., B.L., K.M.) searched their personal records for representative cases of SIS. The operators provided focused and anonymous descriptions of their cases to the leading investigator, who subsequently summarised some key features in a table. Neither personal nor procedural information from the patient, other than the case summary, was entered into any database for sharing among investigators.

The study complied with the principles of good clinical practice and with the Declaration of Hel- sinki for investigation in human beings. Patients provided informed consent for the index intervention and for ulterior data analysis for scientific and quality-control purposes. Due to the retrospective and merely descriptive design of the study, preserving the privacy of personal data, specific informed consent was considered unnecessary by the corresponding review boards and was waived.

\section{Definition of CTO bifurcation lesions}

A bifurcation was defined according to the consensus of the European Bifurcation Club as a significant division of a major epicardial coronary artery, with a relevant side branch (SB) [7, 8]. A relevant $\mathrm{SB}$ was operationally defined as a vessel that the operator wants to preserve $[7,8]$ or $>2.5 \mathrm{~mm}$ in diameter [9]. Likewise, the operator could appoint the arteries as main vessel (MV) or SB according to anatomical or functional criteria $[7,8]$. A CTO-BFL was defined as a bifurcation with at least one of its segments (proximal MV, distal MV or SB) being totally occluded, i.e., $100 \%$ stenosis with distal Thrombolysis In Myocardial Infarction (TIMI) 0 flow for more than 3 months [10]. Any CTO presenting $\leq 10 \mathrm{~mm}$ away from the carina can be adjudicated to the corresponding segment of that bifurcation.

\section{Definition of subintimal shift}

Subintimal shift was defined as complete occlusion or worsening $\geq 30 \%$ diameter stenosis (DS) of a segment of the CTO-BFL, occurring after extraplaque balloon or stent expansion at a different segment of the CTO-BFL. Intracoronary imaging was generally recommended for the diagnosis of SIS, as this phenomenon typically occurs when both wires lye on different anatomic planes (one extraplaque, the other intraplaque) at some point of the bifurcation, considering wires placed in the true lumen or absent as intraplaque.

In case intracoronary imaging were not available to confirm the mechanism, the diagnosis of SIS could be done by means of indirect angiographic signs if the following 3 criteria were met:

- Intentional or accidental extraplaque tracking near the bifurcation ( $5 \mathrm{~mm}$ distal or proximal to the carina). Re-entry at the bifurcation level was considered as potential extraplaque tracking by default;

- Occlusion or worsening $\geq 30 \%$ DS of a segment of the CTO-BFL, triggered by expansion of balloon or stent at a different segment. In case of totally occluded MV or SB at the beginning of the procedure, the worsening $\geq 30 \%$ DS was 


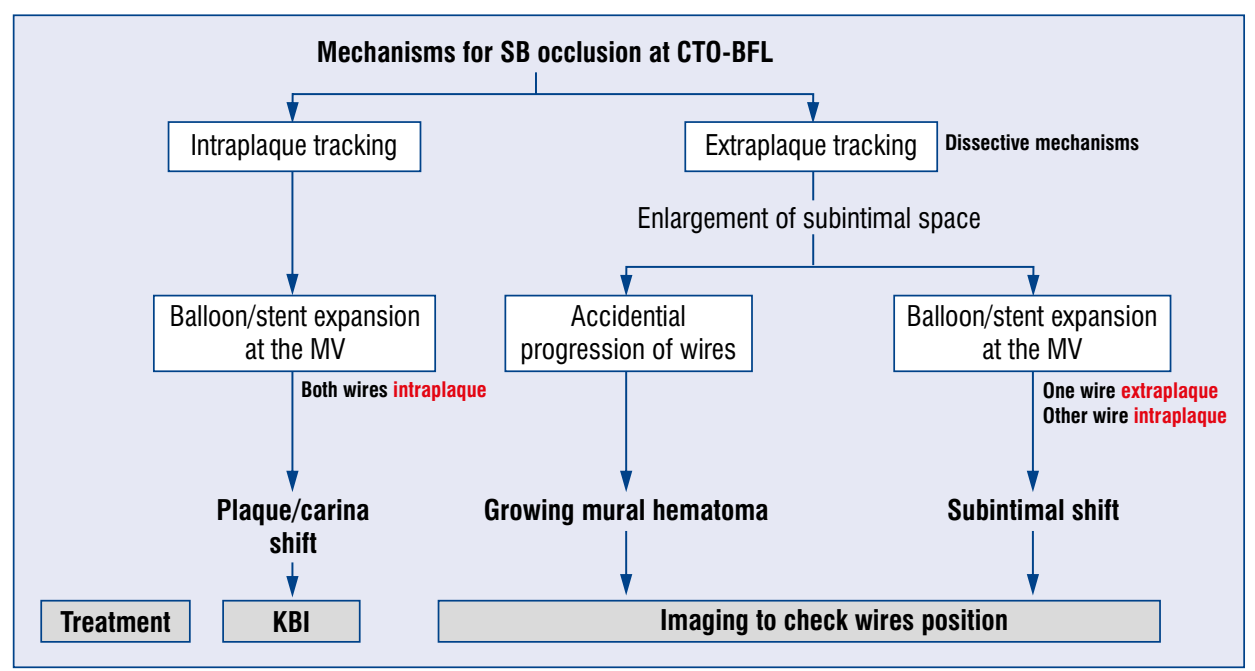

Figure 1. Pathogenic mechanisms for side branch (SB) occlusion at a chronic total occlusion-bifurcation lesion (CTO-BFL). Wires placed in the true lumen or absent will be considered intraplaque; KBI — kissing-balloon inflation; $\mathrm{MV}$ - main vessel.

replaced in the definition by inability to restore antegrade flow after appropriate ballooning;

- Rule out other mechanisms of MV or SB occlusion, like plaque/carina shift (if both wires were intraplaque) or growing mural hematoma due to accidental extraplaque progression of a wire from MV to SB or vice versa (Fig. 1).

Besides the angiographic criteria for diagnosis of SIS, confirmatory and warning signs were as follows:

- Warning signs: 1) presence of a linear filling defect after ballooning and 2) tissue separation in between two balloons during kissing-balloon inflation. These warning signs herald the occurrence of SIS and indicate the need for intravascular imaging prior to stent deployment to prevent MV or SB occlusion (aborted SIS);

- Confirmatory sign: Smooth balloon progression and expansion at the occluded branch, but resulting in suboptimal restoration of the flow (refractoriness to ballooning). This sign enables the differential diagnosis with classical plaque/carina shift, wherein the balloon crosses the occluded ostium with tactile feedback of friction and expands against resistance.

\section{Results}

Following representative cases of SIS were provided by the study investigators.

\section{Case 1}

A 54-year-old male patient with exertional angina, Canadian Cardiac Society (CCS) type II, of approximately 3 years of evolution and large exertion-induced anterior perfusion defect in the scintigraphy underwent coronary angiography, showing a CTO of the ostial left anterior descending (LAD) artery with ambiguous cap (Fig. 2A). Rentrop 3, CC2 septal collaterals were provided by the right coronary artery (RCA) and several septal collaterals were identified as suitable for intervention (Fig. 2B). A retrograde approach with intravascular ultrasound (IVUS) - guided intraplaque tracking was chosen as the primary strategy. A Corsair Pro microcatheter (Asahi Intecc, Aichi, JP) could be easily advanced up to the distal segment of the CTO at the mid LAD. Then an Ultimate wire (Asahi Intecc, Aichi, JP) smoothly advanced up to the left main (LM) before setting up the IVUS, entering the true lumen and the antegrade guiding catheter (Fig. 2C). An IVUS pullback from the circumflex (LCx) showed a short extraplaque course of the retrograde LAD wire (Fig. 3A), with re-entry into the true lumen at the polygon of confluence of the bifurcation (Fig. 3B). Predilation with a $2 \mathrm{~mm}$ balloon was performed at the ostium of the LAD, restoring antegrade TIMI-3 flow in all vessels and showing a linear filling defect (warning sign) at the proximal LAD (Fig. 2D). The operator decided to adjust the stent to the ostium of the $\mathrm{LAD}$, in an attempt to simplify the inter- 


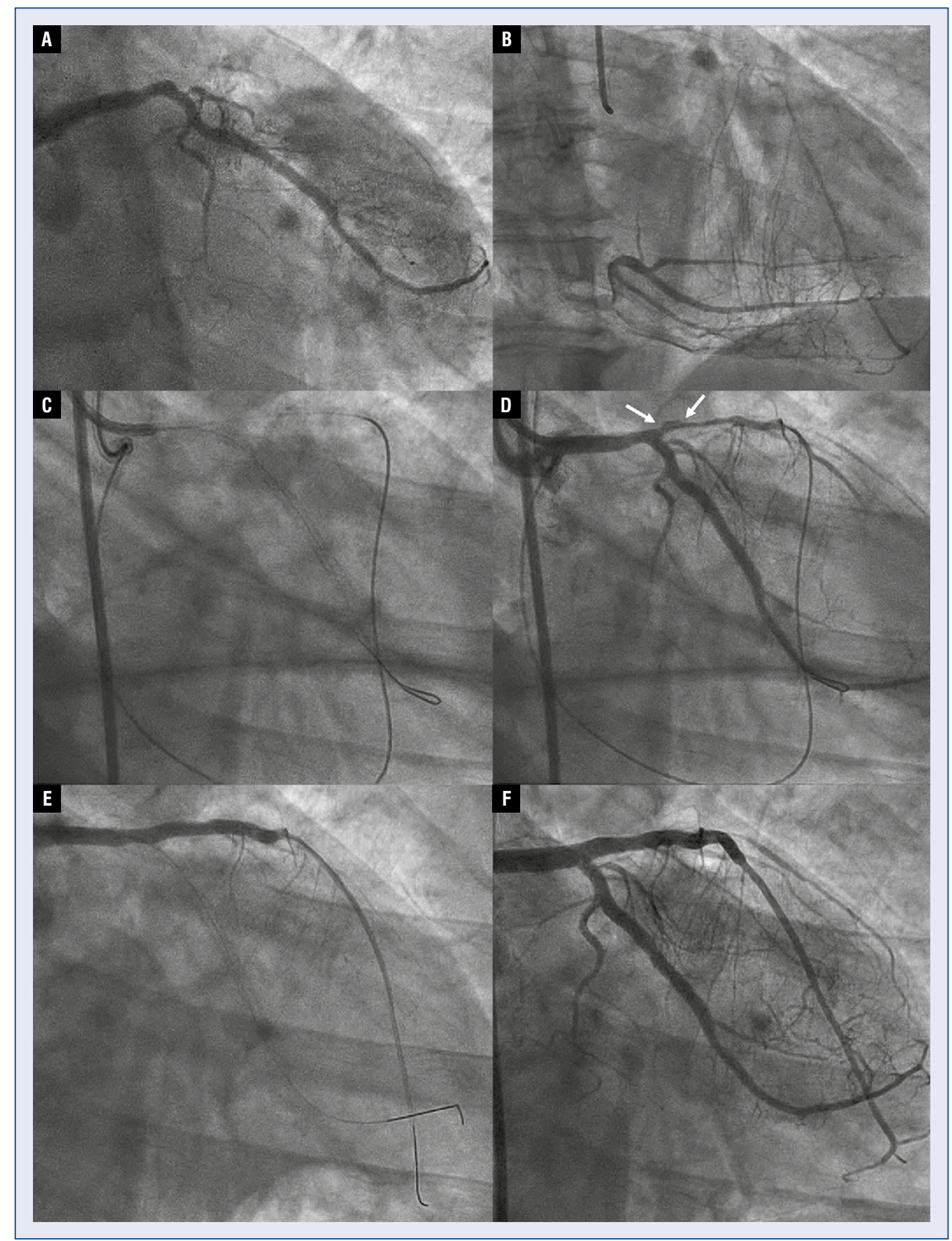

Figure 2. Angiographic summary of case 1. Chronic total occlusion of the ostial left anterior descending coronary artery (LAD) with ambiguous cap (A). Rentrop 3, CC2 septal collaterals from right coronary artery, some of them suitable for intervention (B). Externalization of a retrograde Ultimate wire (Asahi Intecc) into the antegrade guiding catheter (C). Restoration of antegrade TIMI-3 flow in all vessels after predilation of the proximal LAD with a $2 \mathrm{~mm}$ balloon (D), shows a warning sign in proximal LAD (D, arrows). A Xience (Abbott Vascular) $3.0 \times 33 \mathrm{~mm}$ stent was deployed at the ostial LAD, adjusted to the ostium, but the stent deployment resulted in complete occlusion of the left circumflex coronary artery (LCX) (E), refractory to several attempts of ballooning. Stenting of the ostial LCX and the left main coronary artery with an inverse T-stenting technique, followed by kissing-balloon inflation, restored TIMI-3 flow in all vessels with optimal angiographic result (F).

vention, avoiding the involvement of the LM, so a Xience $3.0 \times 33 \mathrm{~mm}$ stent (Abbott Vascular, Santa Clara, CA, USA) was deployed at the LAD ostium.
However, the complete occlusion of the LCx ensued stent deployment (Fig. 2E), refractory to several attempts of ballooning (confirmatory sign). IVUS 


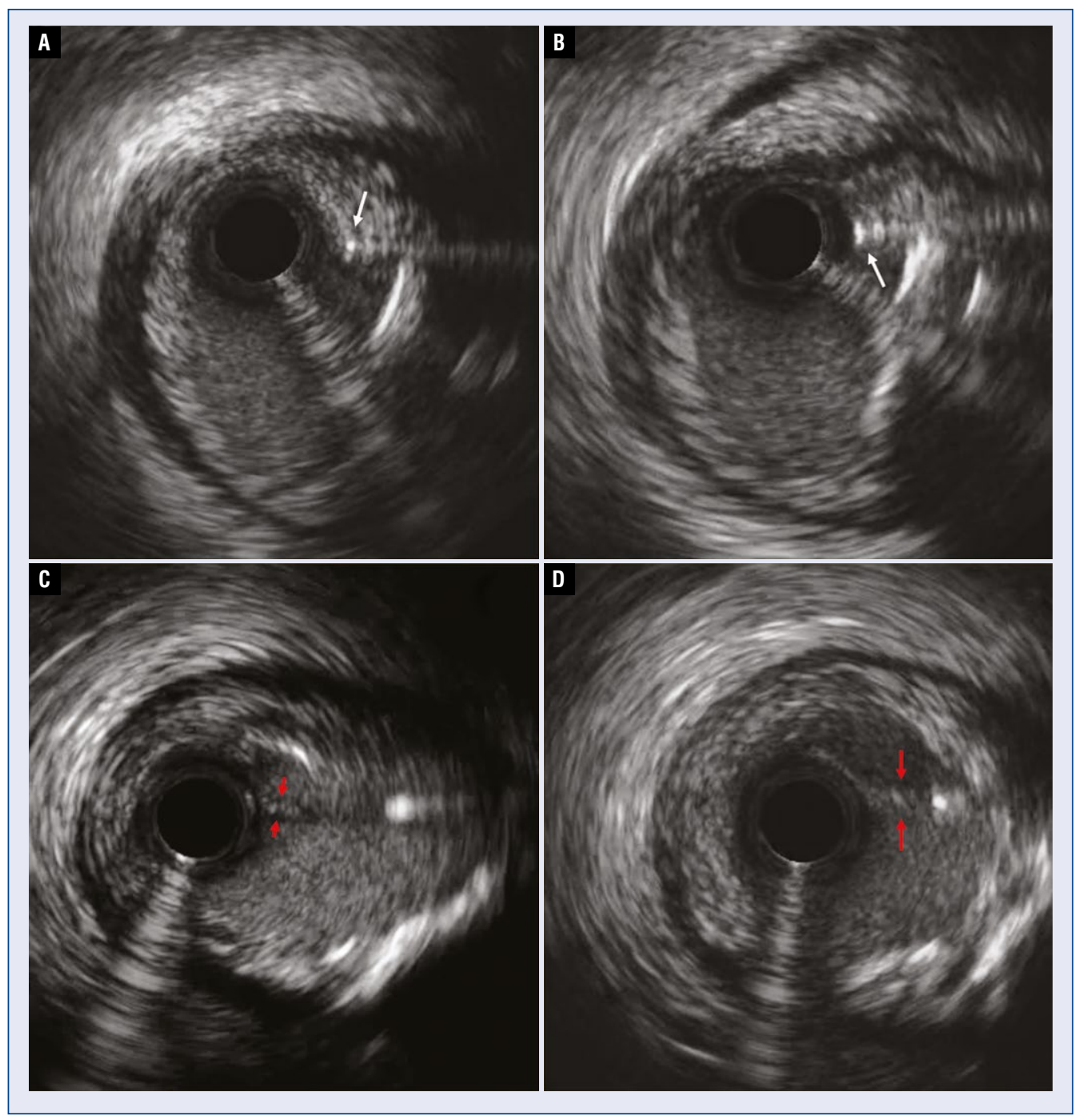

Figure 3. Intravascular ultrasound (IVUS) of case 1. IVUS pullback from the left circumflex coronary artery (LCX) after externalization of the retrograde wire (A, B). A short extraplaque course of the retrograde Ultimate wire $(\mathbf{A}$, white arrow) can be seen at the ostial left anterior descending coronary artery (LAD), with re-entry into the true lumen at the polygon of confluence of the bifurcation (B). IVUS pullback from the LCx after stent deployment (lower panels). Intimomedial flap (subinitmal shift phenomenon) at the ostium of the LCx (C), extending up to the distal left main coronary artery (D), suggesting proximal extension of the LCx, leading to occlusion of the LCx. Notice the LAD wire at 3 o'clock (C, D).

from LCx showed an intimomedial flap at the ostium of the LCx (Fig. 3C, D), thus corroborating SIS as a mechanism for the LCx occlusion (Fig. 4). An optical coherence tomography (OCT) pullback from the $\mathrm{LAD}$ portrayed the collapse of the true lumen in a short segment at the proximal tip of the stent and the extension of dissection plane proximally, involving the ostium of the LCx (Fig. 5). Stenting of the ostial LCx and the LM with an inverse T-stenting technique, followed by kissing-balloon inflation, restored TIMI-3 flow in all vessels with optimal angiographic result (Fig. 2F). The final OCT nicely depicts the true lumen sandwiched in the neocarina (Fig. 6).

\section{Case 2}

A 74-year-old male with prior coronary artery bypass grafting, namely internal mammary artery to LAD and saphenous vein graft (SVG) to the posterior descending artery (PDA), presented angina CCS II and inferior ischemia in scintigraphy. Angiography showed occlusion of the SVG and the patient was referred for CTO PCI of the RCA after a failed attempt in another center. The CTO of the RCA started in the mid segment, where a remaining dissection from the previous $\mathrm{PCI}$ attempt was observed (Fig. 7A) and extended beyond the crux cordis into both the PDA and the postero- 


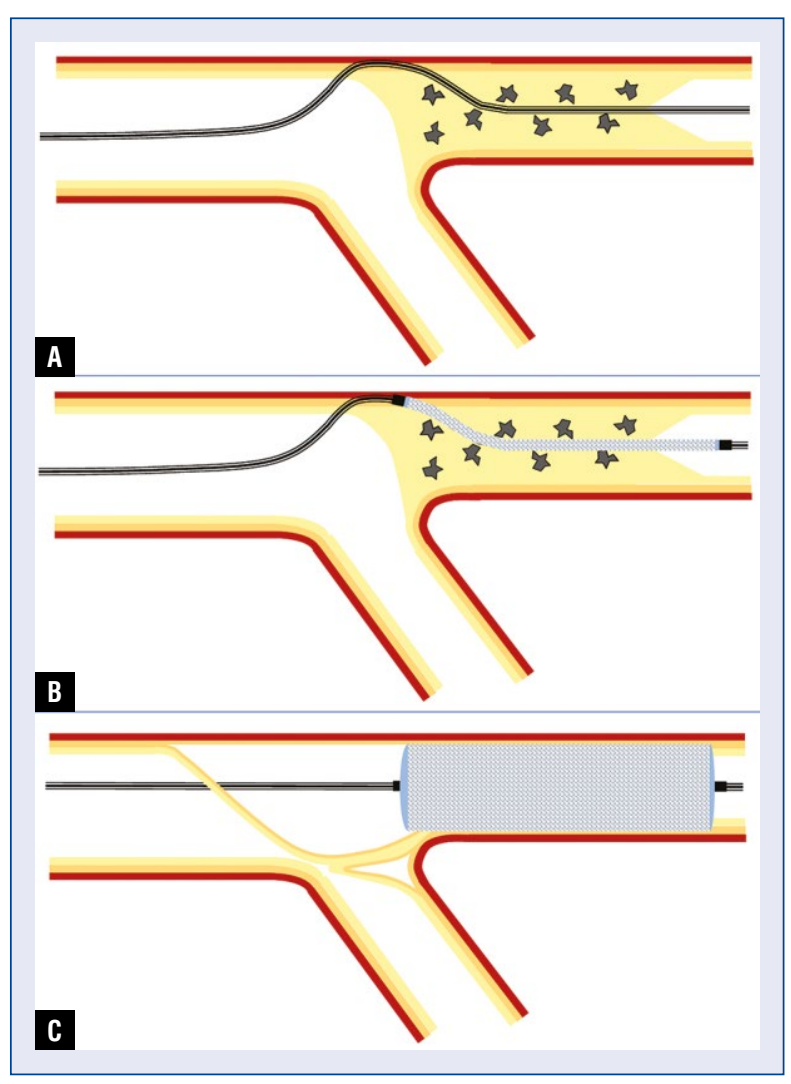

Figure 4. Mechanism of subintimal shift. The scheme illustrates the representative case 1 . The retrograde wire followed a short subintimal course at the ostial left anterior descending coronary artery (LAD), but reentering into the true lumen at the polygon of confluence of the bifurcation (A). The stent was adjusted to the LAD ostium, thus not covering the entry (i.e., proximal) point to the subintimal space (B). The stent expansion resulted in proximal extension of the subintimal space, occluding the ostium of the left circumflex coronary artery (LCX) (C). Although balloons could be easily inflated at the ostium of the LCx, it was repeatedly ineffective, because elastic recoil of the flap immediately followed balloon deflation.

lateral coronary artery (PLA) (Fig. 7B). Septal CC2 collaterals were provided by the LAD (Fig. 7B). A retrograde approach through the native LAD was primarily chosen, reaching the distal PDA through a septal collateral with a Corsair Pro microcatheter over a Sion black wire (Asahi Intecc, Aichi, JP) (Fig. 7C). At this point, a Fielder XT-R wire (Asahi Intecc, Aichi, JP) was knuckled and progressed from the antegrade gear up to the PLA, supported by a Turnpike microcatheter and a 7 Fr-Guideliner extension (Teleflex, Wayne, USA) (Fig. 7D). Then the distal cap of the CTO was retrogradely punctured at the PDA with a Hornet 14 wire (Boston scien- tific, Marlborough, USA), followed by retrograde progression of a knuckled Fielder XT wire (Asahi Intecc, Aichi, JP) over the bifurcation until successful externalization by means of reverse controlled antegrade and retrograde tracking (rCART) and tip-in techniques [11]. Kissing balloon inflation at the crux cordis showed separation of the balloons due to the interposition of an intimomedial flap, which is considered a warning sign of impending SIS phenomenon (Fig. 7E). An IVUS pullback confirmed that the wires at each vessel of the bifurcation were at different anatomic planes: the antegrade PLA wire was extraplaque, while the retrograde PDA wire was intraplaque (Fig. 8). This is the anatomic condition for SIS to occur if the stent were deployed at PLA. To avoid this complication the true lumen of PLA was successfully rewired under IVUS-guidance, through a dual-lumen microcatheter over the PDA wire. Provisional T-stenting was finally performed with excellent angiographic result (Fig. 6F).

\section{Case 3}

A 59-year-old male patient with CCS II angina and positive scintigraphy in the lateral wall, showed CTO of the ostial LCx and first diagonal branch (D1) in angiography, the latter with an ambiguous cap (Fig. 9A, B). The patient was referred for PCI of both CTO lesions. After successful antegrade revascularization of the $\mathrm{LCx}$, a retrograde approach was preferred for the ostial D1 because of its cap ambiguity. A Caravel microcatheter reached the distal D1 over a Suoh 03 wire (Asahi Intecc, Aichi, JP), through an epicardial collateral stemming from the PLA and the distal cap was punctured using a Gaia $3^{\text {rd }}$ wire (Asahi Intecc, Aichi, JP). Then a Fielder XT wire was knuckled up to the proximal LAD (Fig. 9C, D), where retrograde kissing-wire was successfully performed with Confianza pro 12 (Asahi Intecc, Aichi, JP), thus enabling the externalization. After pre-dilatation with a $2.5 \mathrm{~mm} \mathrm{NC}$ balloon a $2.5 \times 24 \mathrm{~mm}$ Promus Premier stent (Boston scientific, Marlborough, USA) was adjusted to the D1 ostium (Fig. 9E). The stent deployment triggered SIS, severely compromising the proximal LAD (Fig. 9F). The proximal LAD was successfully rewired, using a Confianza pro 12 (Asahi Intecc, Aichi, JP) to perforate the intimomedial flap (Fig. $9 \mathrm{G}$ ), followed by provisional T-stenting and final kissing balloon dilatation (Fig. 9H), with excellent angiographic result (Fig. 9I).

\section{Summary of the series}

Four additional paradigmatic cases are described in full detail in the Supplementary mate- 


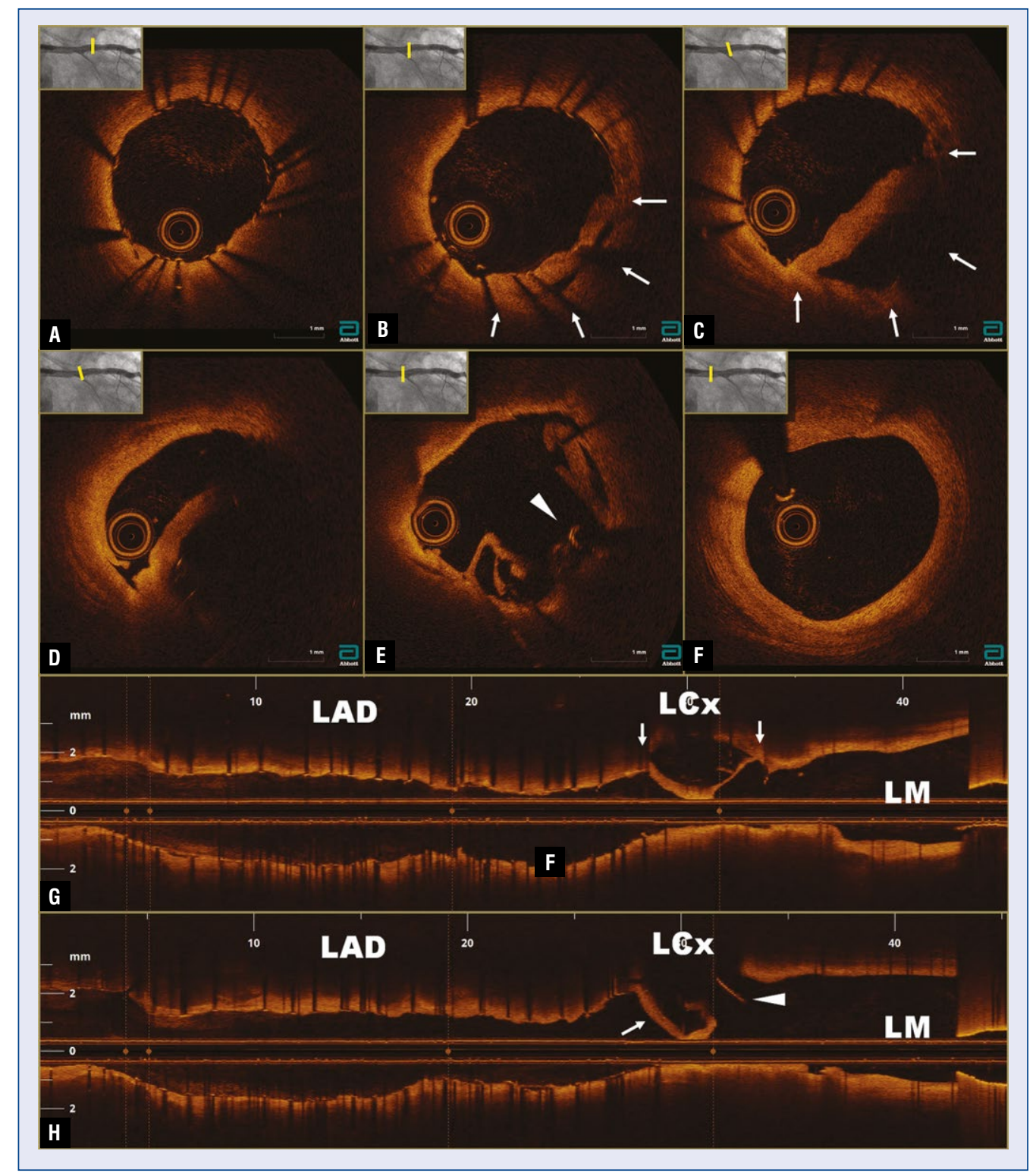

Figure 5. Optical coherence tomography from the left anterior descending coronary artery (LAD) after stent expansion in case 1. Pullback from distal $(\mathbf{A})$ to proximal $(\mathbf{F})$ shows the stent deployed distally at the true lumen $(\mathbf{A})$, but at the false lumen at the proximal stent tip, with the true lumen collapsed against the vessel wall (B, white arrows). An intimomedial flap (subintimal shift phenomenon) (C, D) acts as flow-divider between the true lumen (white arrows) going to the left circumflex coronary artery (LCX) and the false lumen going to the stent at LAD. The wire at the LCX (E, arrowhead) connects the true lumen of left main coronary artery (LM) (F) with the true lumen of LCx. The longitudinal view shows the intimomedial flap occluding the ostium of the LCx (G, white arrows), with the wire (H, arrowhead) connecting the true lumen of LM and LCx.

rial. Table 1 summarises the key features of the whole series $(n=7)$. All cases met the hereby proposed angiographic criteria and two were confirmed by means of intracoronary imaging ( 2 by IVUS, 1 by OCT). Three (42.9\%) cases were preceded by warning signs in angiography. Five $(71.4 \%)$ cases were triggered by stent expansion, $1(14.3 \%)$ was triggered by balloon expansion and $1(14.3 \%)$ case was aborted after noticing an angiographic warning sign, with subsequent confirmation in IVUS. The angiographic expression was total occlusion in $4(57.1 \%)$ cases and in 2 of them it was decided not to rescue the occluded branch, subsequently resulting in a rise of cardiac markers $>70 \times$ upper limit of normality, thus failing to meet criteria for technical or procedural success [9]. Four (57.1\%) 


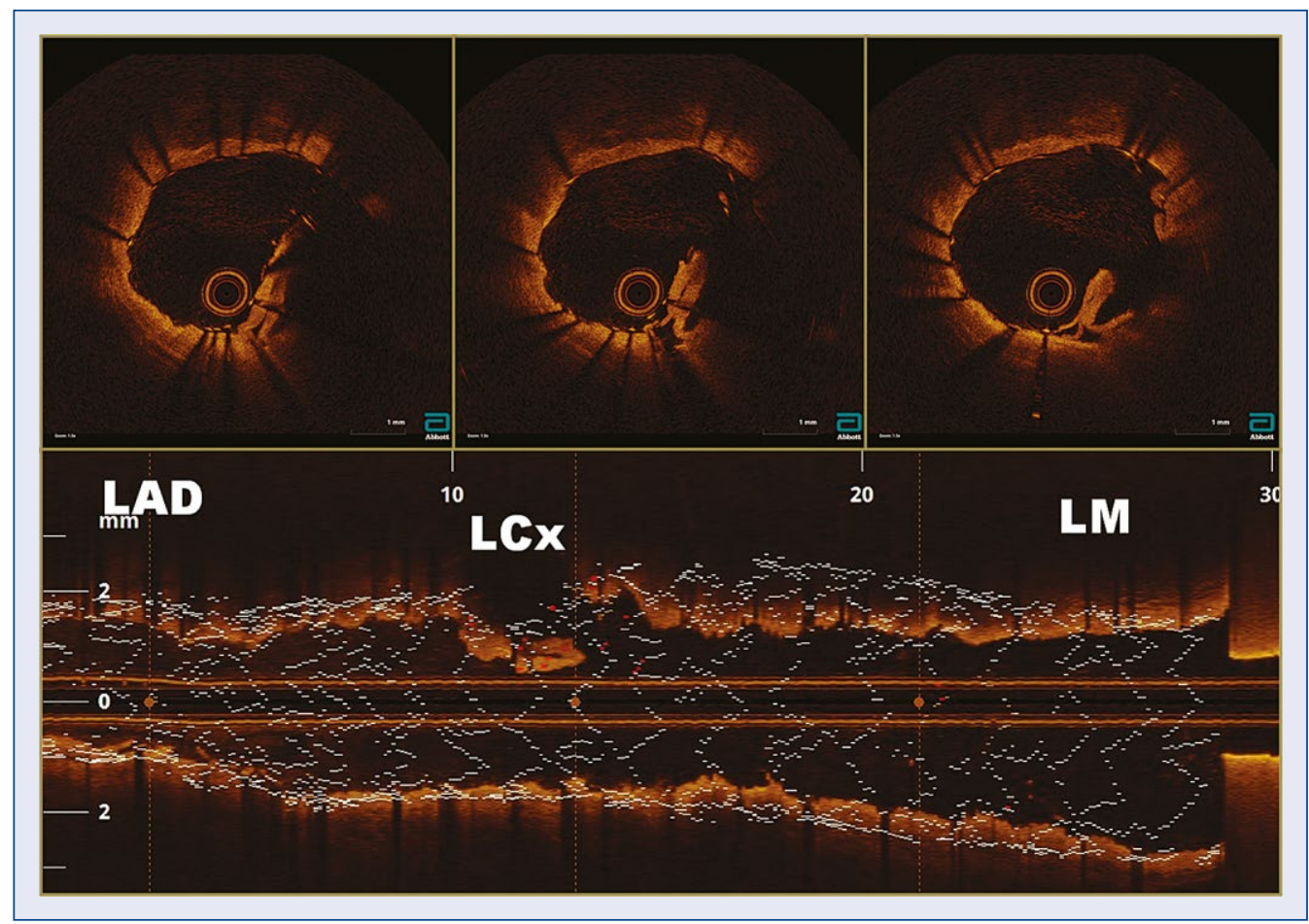

Figure 6. Optical coherence tomography from the left anterior descending coronary artery (LAD) after T-stenting of left circumflex coronary artery (LCX) in the bifurcation. Pullback from distal (left) to proximal (right) nicely depicts the true lumen sandwiched in the neocarina; LM - left main coronary artery.

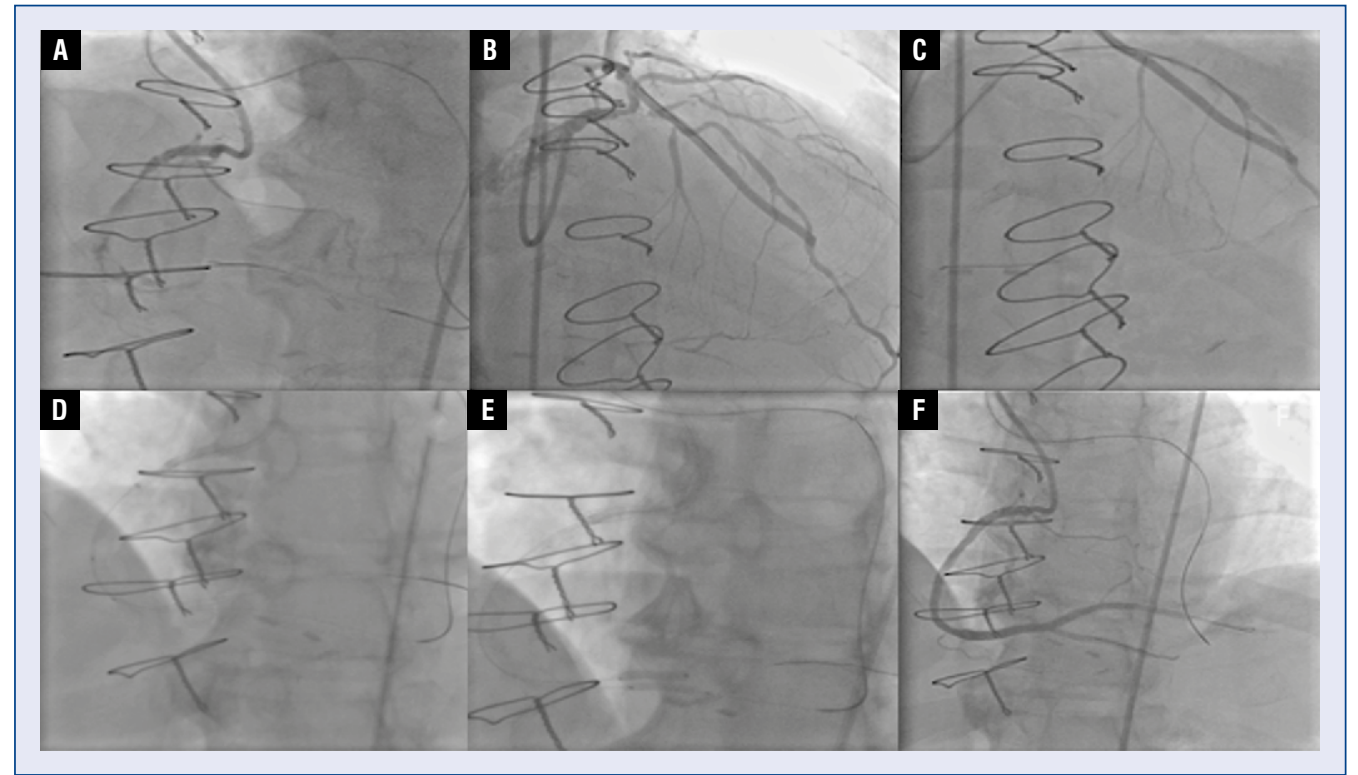

Figure 7. Angiographic summary of case 2. Chronic total occlusion of the mid right coronary artery (A), extending beyond the crux cordis into both the posterior descending artery (PDA) and posterior left ventricular branch (PLA) (B). Septal collaterals CC2 from the left anterior descending coronary artery to the PDA (B). Septal wiring by Sion black Wire and Corsair Pro (Asahi Intecc, Aichi, JP) (C). Antegrade wiring of the PLA branch by a knuckled Fielder XT-R (Asahi Intecc, Aichi, JP) and externalization of the retrograde wire (D). Kissing balloon inflation demonstrates a warning sign of subintimal shift if the stent were deployed, namely, the excessive separation between balloons during inflation (E), pointing out the presence on an intimo-medial flap interposed between them. Final angiographic result after intravascular ultrasound-guided rewiring of the PLA and T-stenting (F). 


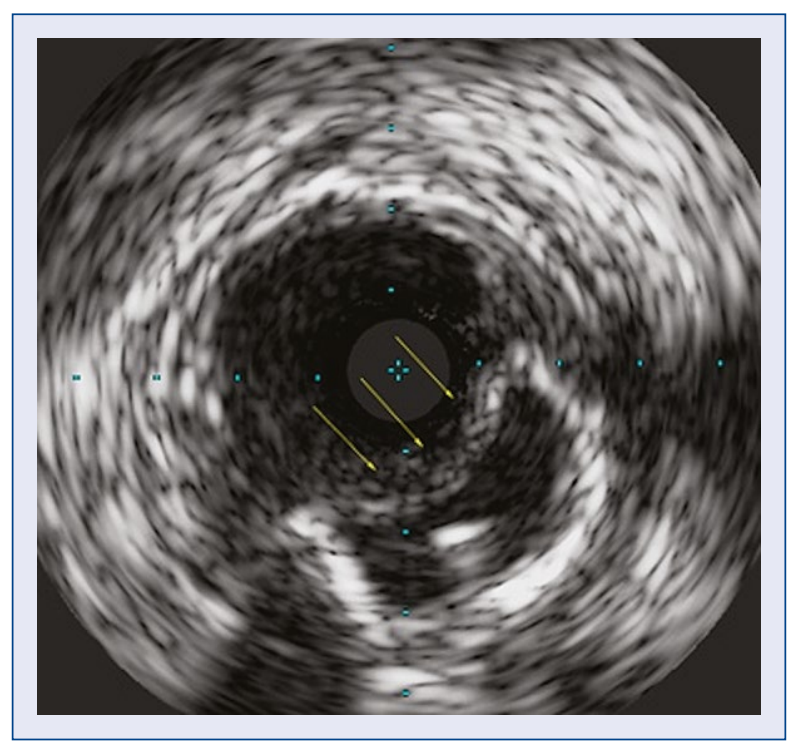

Figure 8. Intravascular ultrasound (IVUS) image of case 2. IVUS pullback from the posterior left ventricular branch (PLA) demonstrates the intimo-medial flap at the bifurcation (arrows), with each wire lying on a different plane: PLA wire extraplaque, posterior descending artery wire intraplaque (true lumen).

cases required bailout intervention on the compressed segment and one additional case did not require more intervention than preventive wiring of the branch. All SIS cases initially treated with balloon dilatation, were refractory to ballooning (confirmatory sign).

\section{Discussion}

Side branch occlusion during CTO PCI is associated with adverse outcomes [4] and it is essential for the interventional cardiologist to understand the mechanism underneath this complication to efficiently solve it. In case of pure intraplaque tracking of wires and devices, classical plaque/ /carina shift is a plausible pathogenic mechanism, like in non-CTO-BFL (Fig. 1). The likelihood of plaque-shift in CTO-BFL is not negligible, as plaque burden is usually large, but this mechanism is easy to recognize: during the rescue of the occluded SB, the balloon crosses the occluded ostium under tactile feedback of friction, expands against resistance and the inflation usually improves the distal flow at the rescued SB, like in non-CTO-BFL.

Conversely, in case of extraplaque tracking, other mechanisms of SB compression are more likely to occur than classical plaque shift, because the expansion of balloons or stents will tend to accommodate into the elastic subintimal space, thus inflicting less severe alteration of geometry to the plaque mass (Fig. 1). Extraplaque tracking has been documented with intracoronary imaging in more than $25 \%$ of cases wherein a true-to-true lumen approach was initially reported $[12,13]$. If it occurs in the vicinity of a CTO-BFL, the extraplaque expansion of balloons or stents may result in propagation of the dissection plane over the bifurcation and shift of the subintimal space from $\mathrm{MV}$ to $\mathrm{SB}$, or vice versa. This mechanism is named SIS and is a potentially serious complication, generally leading to SB occlusion. According to available research, this is the first description of the "subintimal shift" - phenomenon.

IVUS and OCT provide definite evidence about SIS, showing the extension of the subintimal flap into the occluded branch. However, these imaging modalities are not always available and they can even be contraindicated at certain stages of the CTO intervention. SIS can be then diagnosed with standard angiography, following the criteria hereby proposed. Angiographic warning signs, like the peri-bifurcational linear filling defect after ballooning (Figs. 2D; 9F, G) or the excessive separation between balloons while kissing (Fig. 7E), can be instrumental to reconduct the intervention, but they might be missing or unnoticed in many cases. In the present series, only 3 out of 7 cases showed a warning sign in angiography. Therefore, the use of intravascular imaging, preferably IVUS, is encouraged if a peri-bifurcational extraplaque tracking is suspected; to understand the tissue planes, to guide the repositioning of the wires or to undertake alternative measurements to avoid branch occlusion. The key practical tip is keeping in mind that SIS may occur when the wires of each bifurcation vessel are placed at different anatomic planes at some point of the bifurcation: one intraplaque, the other extraplaque. Thereafter the aim will be repositioning them at the same plane, whenever possible. SIS will not happen if both wires are intraplaque or if both wires are extraplaque, even though the latter scenario might intuitively seem distressing.

Once SIS occurs, the complication has some peculiarities that might seem counterintuitive from the perspective of non-CTO bifurcational interventions and might lead to inefficient manoeuvres and stagnation of the procedure, if ignored. The most counterintuitive feature is the refractoriness to ballooning. While in non-CTO bifurcational PCI the occlusion of a SB immediately triggers a reaction of the operator, who will balloon the occluded 


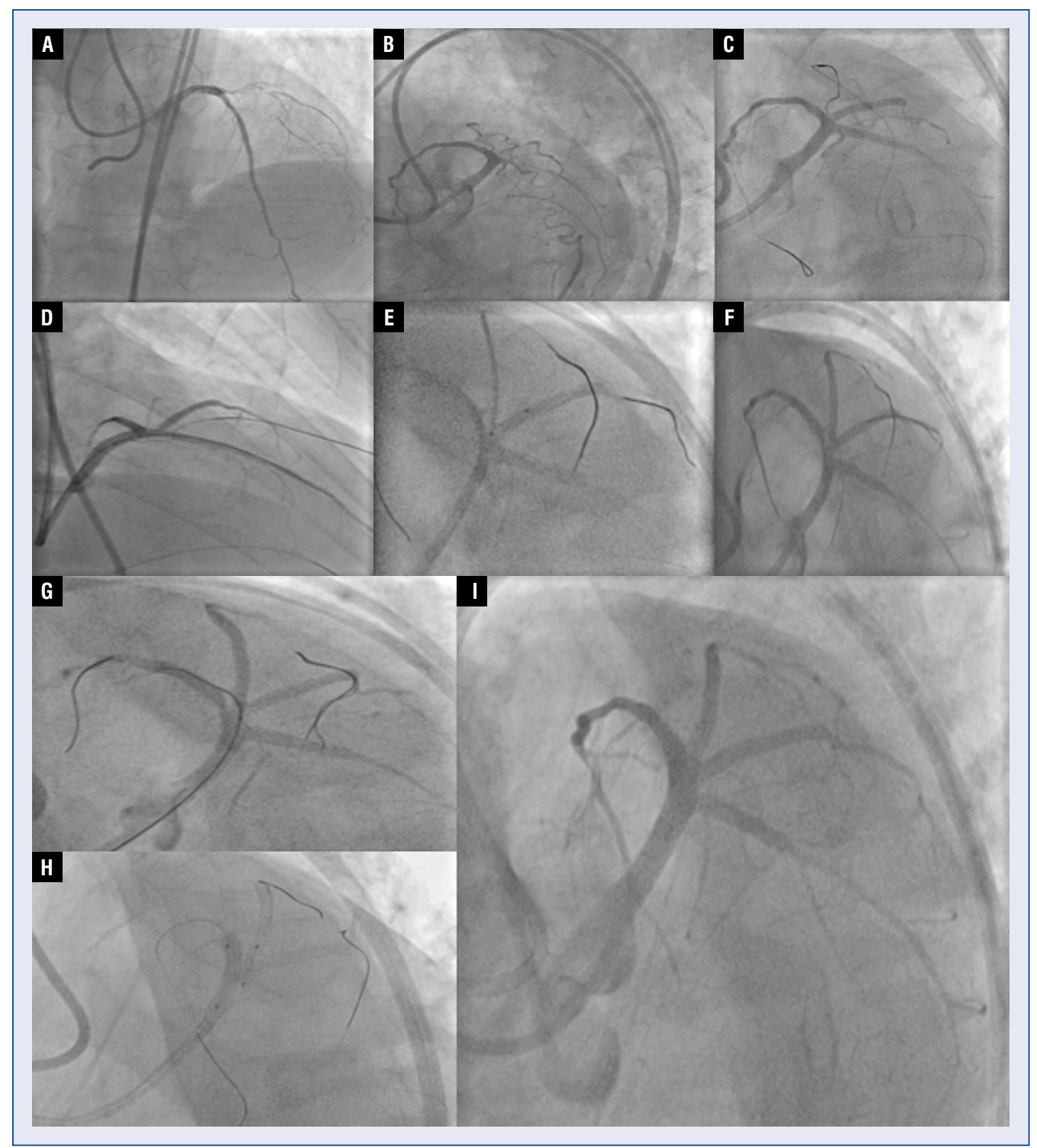

Figure 9. Angiographic summary of case 3. Chronic total occlusion (CTO) of the proximal left circumflex coronary artery and ostial first diagonal branch (D1) with ambiguous cap (A, B). Retrograde approach for the CTO of D1, using a Caravel microcatheter over a Suoh 03 wire (Asahi intecc), followed by puncture of the distal cap with a Gaia $3^{\text {rd }}$ wire and extraplaque tracking with a Fielder XT wire (Asahi Intecc, Aichi, JP) (C, D). After retrograde cap penetration with Confianza Pro 12 wire (Asahi Intecc, Aichi, JP) and externalization by tip-in manoeuvre, a stent was adjusted to the ostium of D1 (E). After stent deployment, subintimal shift severely compromised proximal left anterior descending (LAD) (F). Intimal rewiring of the proximal LAD with a Confianza pro 12 wire (Asahi Intecc) (G). After the T-stenting technique and final kissing-balloon (H) an excellent angiographic result was achieved (I).

vessel to reopen it, this manoeuvre will be useless in most cases of SIS (all cases wherein ballooning was attempted, in this series). The balloon will fully expand without resistance, but after deflation, the intimo-medial flap will recoil again, bringing the operator back to the starting point. Direct stenting will restore antegrade flow and will spare time. In case of total occlusion, a preliminary check with IVUS can be reassuring to rule out other complica- tions and to properly size the stent to implant. In other cases, aggressive interventional manoeuvres, like the puncture of the true lumen through the occluding intimo-medial flap with stiff tapered wires, might be required to rewire the occluded branch (case 3).

It is currently debatable whether covering the entry and exit points of the extraplaque course with the firstly deployed stent might prevent or 
Table 1. Summary of key points in the case series.

\begin{tabular}{|c|c|c|c|c|c|c|c|}
\hline & Case 1 & Case 2 & Case 3 & Case 4 & Case 5 & Case 6 & Case 7 \\
\hline & \multicolumn{7}{|c|}{ Bifurcation location } \\
\hline & $\begin{array}{l}\text { LM-LAD (MV) } \\
\text { LCx (SB) }\end{array}$ & $\begin{array}{c}\text { RCA (MV) } \\
\text { - PD-PL } \\
\text { (SB) }\end{array}$ & $\begin{array}{l}\text { LAD (MV) } \\
- \text { ICA (SB) }\end{array}$ & $\begin{array}{l}\text { RCA (MV) } \\
- \text { PD-PL } \\
\text { (SB) }\end{array}$ & $\begin{array}{c}\text { RCA (MV) } \\
\text { RV (SB) }\end{array}$ & $\begin{array}{c}\text { RCA (MV) } \\
\text { RV (SB) }\end{array}$ & $\begin{array}{c}\text { RCA (MV) } \\
\text { RV (SB) }\end{array}$ \\
\hline Medina & 010 & 111 & 001 & 100 & 010 & 010 & 010 \\
\hline Approach & Retrograde & Retrograde & Retrograde & Retrograde & Retrograde & Retrograde & Retrograde \\
\hline Warning sign in angio & Yes & Yes & No & No & Yes & No & No \\
\hline Aborted SIS & No & Yes & No & No & No & No & No \\
\hline \multicolumn{8}{|l|}{ Trigger: } \\
\hline Balloon inflation & No & NA & No & No & No & Yes & No \\
\hline Stent expansion & Yes & NA & Yes & Yes & Yes & No & Yes \\
\hline Jeopardised segment & LCx (SB) & PD (DMV) & LAD (MV) & $\begin{array}{c}\mathrm{PL}(\mathrm{SB}) \\
\mathrm{PD}(\mathrm{DMV})\end{array}$ & $\mathrm{RV}(\mathrm{SB})$ & $\mathrm{RV}(\mathrm{SB})$ & $\mathrm{RV}(\mathrm{SB})$ \\
\hline $\begin{array}{l}\text { Angiographic } \\
\text { expression }\end{array}$ & $\begin{array}{c}\text { Total } \\
\text { occlusion }\end{array}$ & $\begin{array}{c}\text { Flow } \\
\text { limiting } \\
\text { dissection }\end{array}$ & $\begin{array}{l}\text { Severe } \\
\text { stenosis }\end{array}$ & $\begin{array}{c}\text { Total } \\
\text { occlusion }\end{array}$ & $\begin{array}{c}\text { Total } \\
\text { occlusion }\end{array}$ & $\begin{array}{l}\text { Severe } \\
\text { stenosis }\end{array}$ & $\begin{array}{c}\text { Total } \\
\text { occlusion }\end{array}$ \\
\hline \multicolumn{8}{|l|}{ Documented by: } \\
\hline Angiography & Yes & Yes & Yes & Yes & Yes & Yes & Yes \\
\hline IVUS & Yes & Yes & No & No & No & No & No \\
\hline OCT & Yes & No & No & No & No & No & No \\
\hline $\begin{array}{l}\text { Need for bailout } \\
\text { intervention in the SB }\end{array}$ & Yes & Yes & Yes & Yes & NA & No & NA \\
\hline $\begin{array}{l}\text { Refractory to } \\
\text { ballooning }\end{array}$ & Yes & NA & Yes & Yes & NA & NA & NA \\
\hline Technical success & Yes & Yes & Yes & Yes & No & Yes & No \\
\hline Procedural success & Yes & Yes & Yes & Yes & No & Yes & No \\
\hline
\end{tabular}

CTO — chronic total occlusion; DMV — distal main vessel; ICA — intermediate coronary antery; IVUS — intravascular ultrasound; LAD — left anterior descending coronary artery; LCx — left circumflex coronary artery; LM — left main coronary artery; MV — main vessel; NA — non applicable; OCT — optical coherence tomography; PD — posterior descending coronary artery; PL - posterolateral coronary artery; $\mathrm{RCA}$ - right coronary artery; RV — right ventricular branch; SB — side branch; SIS — subintimal shift

limit SIS. Some operators argue that the subintimal space would be expanded anyway and the risk of SIS would still exist, with the additional handicap of rewiring the occluded branch through the stent struts across the bifurcation. Nonetheless, other operators argue that the subintimal expansion would be minimized, because coronary balloons (including carrier-balloons of stents) are designed to start their inflation at both tips (Suppl. Video 1), so the expansion of the subintimal space would be thus limited. In our large experience of over 5000 CTO cases, both scenarios have already occurred, but evidence to resolve this controversy should be generated in future studies.

The term SIS was firstly proposed at the European Bifurcation Club, to be consistent with consensus terminology used for bifurcational PCI $[7,8]$. The mechanism of occlusion of side branches during PCI due to the expansion of devices at the
MV was initially named "plaque shift". This term has been recently replaced by the concept of "carina shift", to highlight that the occlusion can simply be a consequence of the altered geometry of the bifurcation anatomy provoked by the interventional sequence [8]. In an analogy with this consensus, the term SIS can be applied to a specific setting of bifurcation in a CTO.

\section{Limitations of the study}

This pilot report is based on a case series. The low recognition of SIS and a lack of standardization has thus far prevented more meaningful scientific designs. This approach was necessary however, and was sufficient in illustrating the concept according to a connoisseurs' experience, but does not reflect the real incidence of the phenomenon and its peculiarities. A systematic assessment in large databases would be required to complete 
the clinical depiction of SIS and outline its clinical importance in the routine of a cathlab.

Although the proposed angiographic criteria are useful to identify the leading mechanism for $\mathrm{SB}$ occlusion in the CTO-BFL, confirmation of SIS is only possible by means of intracoronary imaging, and should be encouraged whenever possible.

\section{Conclusions}

Subintimal shift is a potential complication at the bifurcation level during CTO PCI. Intravascular imaging is recommended to prevent SIS, aiming to understand intra- or extraplaque wire position before stent deployment. There are some counterintuitive peculiarities of this phenomenon, like its refractoriness to ballooning, are certain to be known by CTO operators for its efficient resolution.

\section{Acknowledgments}

The authors thank Ricardo Sanz-Ruiz, Ignacio Amat-Santos and the Cardiology Department of Valladolid University Clinic Hospital for their active support and the organization of the live course in which case 1 was performed.

Conflict of interest: Juan Luis Gutiérrez-Chico received consultancy fees and speaker honoraria from Abbott, Asahi Intecc and Boston Scientific. Bernward Lauer received speaker honoraria and/ /or travel support from Daiichi Sankyo, Asahi Intecc, Novartis, Bayer Health, AstraZeneca, Pfizer BMS, Nicolai, Boston Scientific. Sylvia Otto received travel support and/or speaker honoraria from Daiichi Sankyo, Pfizer BMS, Novartis, Berlin Chemie, Bayer Health, BBraun, Abbott, Boston Scientific. Kambis Mashayekhi received consultancy fees and speaker honoraria from Abbott, Abiomed, Asahi Intecc, AstraZeneca, Biotronik, Boston Scientific, Cardinal Health, Daiichi Sankyo, Medtronic, Schockwave, Teleflex, Terumo. All other authors had nothing to disclose.

\section{References}

1. Konstantinidis NV, Werner GS, Deftereos S, et al. Temporal trends in chronic total occlusion interventions in europe. Circ Cardiovasc Interv. 2018; 11(10): e006229, doi: 10.1161/CIRCINTERVENTIONS.117.006229, indexed in Pubmed: 30354635.

2. Galassi AR, Boukhris M, Tomasello SD, et al. Incidence, treatment, and in-hospital outcome of bifurcation lesions in patients undergoing percutaneous coronary interventions for chronic total occlusions. Coron Artery Dis. 2015; 26(2): 142-149, doi: 10.1097/MCA.0000000000000194, indexed in Pubmed: 25405930.

3. Chen Sl, Ye F, Zhang Jj, et al. Clinical outcomes after recanalization of a chronic total occluded vessel with bifurcation lesions: results from single-center, prospective, chronic total occlusion registry study. Chin Med J (Engl). 2012; 125(6): 1035-1040, indexed in Pubmed: 22613527.

4. Ojeda S, Pan M, Gutiérrez A, et al. Bifurcation lesions involved in the recanalization process of coronary chronic total occlusions: Incidence, treatment and clinical implications. Int J Cardiol. 2017; 230: 432-438, doi: 10.1016/j.ijcard.2016.12.088, indexed in Pubmed: 28041711.

5. Baystrukov VI, Kretov EI, Boukhris M, et al. A randomized trial of bifurcation stenting technique in chronic total occlusions percutaneous coronary intervention. Coron Artery Dis. 2018; 29(1): 30-38, doi: 10.1097/MCA.0000000000000551, indexed in Pubmed: 29220345.

6. Guan C, Yang W, Song L, et al. Association of acute procedural results with long-term outcomes after CTO PCI. JACC Cardiovasc Interv. 2021; 14(3): 278-288, doi: 10.1016/j.jcin.2020.10.003, indexed in Pubmed: 33541539.

7. Louvard Y, Thomas M, Dzavik V, et al. Classification of coronary artery bifurcation lesions and treatments: time for a consensus! Catheter Cardiovasc Interv. 2008; 71(2): 175-183, doi: 10.1002/ ccd.21314, indexed in Pubmed: 17985377.

8. Lassen JF, Holm NR, Banning A, et al. Percutaneous coronary intervention for coronary bifurcation disease: 11th consensus document from the European Bifurcation Club. EuroIntervention. 2016; 12(1): 38-46, doi: 10.4244/EIJV12I1A7, indexed in Pubmed: 27173860.

9. Ybarra LF, Rinfret S, Brilakis ES, et al. Definitions and clinical trial design principles for coronary artery chronic total occlusion therapies: CTO-ARC consensus recommendations. Circulation. 2021; 143(5): 479-500, doi: 10.1161/CIRCULATIONAHA.120.046754, indexed in Pubmed: 33523728.

10. Galassi AR, Werner GS, Boukhris M. Percutaneous recanalisation of chronic total occlusions: 2019 consensus document from the EuroCTO Club. EuroIntervention. 2019; 15: 198-208.

11. Mashayekhi K, Valuckiene Z, Neuser H, et al. Wire externalisation techniques for retrograde percutaneous coronary interventions of chronic total occlusions. EuroIntervention. 2017; 13(12): e1489-e1490, doi: 10.4244/EIJ-D-16-00009, indexed in Pubmed: 29208580.

12. Song L, Maehara A, Finn MT, et al. Intravascular ultrasound analysis of intraplaque versus subintimal tracking in percutaneous intervention for coronary chronic total occlusions and association with procedural outcomes. JACC Cardiovasc Interv. 2017; 10(10): 1011-1021, doi: 10.1016/j.jcin.2017.02.043, indexed in Pubmed: 28521919.

13. Walsh SJ, Hanratty CG, McEntegart M, et al. Intravascular healing is not affected by approaches in contemporary CTO PCI: the CONSISTENT CTO study. JACC Cardiovasc Interv. 2020; 13(12): 1448-1457, doi: 10.1016/j.jcin.2020.03.032, indexed in Pubmed: 32553333. 